\title{
Differences in atheroma between Caucasian and Asian subjects with anterior stroke: A vessel wall MRI study
}

\author{
Hiroko Watase (D) , ${ }^{1}$ Mi Shen, ${ }^{2}$ Binbin Sui,, ${ }^{3}$ Peiyi Gao, ${ }^{2,4}$ Dong Zhang, ${ }^{5}$ Jie Sun, ${ }^{6}$ \\ Niranjan Balu, ${ }^{6}$ Daniel S Hippe, ${ }^{6}$ Gail P Jarvik, ${ }^{7}$ Xihai Zhao, ${ }^{8}$ Rui Li, ${ }^{8}$ Shuo Chen, ${ }^{8}$ \\ Chun Yuan, ${ }^{6}$ Thomas S Hatsukami ${ }^{1}$
}

To cite: Watase $\mathrm{H}$, Shen $\mathrm{M}$, Sui B, et al. Differences in atheroma between Caucasian and Asian subjects with anterior stroke: A vessel wall MRI study. Stroke \& Vascular Neurology 2021;6: e000370. doi:10.1136/ svn-2020-000370

- Additional material is published online only. To view please visit the journal online (http://dx.doi.org/10.1136/svn2020-000370).

Received 3 March 2020 Revised 20 May 2020

Accepted 17 June 2020 Published Online First 13 August 2020

\section{Check for updates}

(c) Author(s) (or their employer(s)) 2021. Re-use permitted under CC BY-NC. No commercial re-use. See rights and permissions. Published by BMJ.

For numbered affiliations see end of article.

Correspondence to Dr Thomas S Hatsukami; tomhat@uw.edu

\section{ABSTRACT}

Background and purpose While extracranial carotid artery stenosis is more common among Caucasians and intracranial artery stenosis is more common among Asians, the differences in atherosclerotic plaque characteristics have not yet been extensively examined. We sought to investigate plaque location and characteristics within extracranial carotid and intracranial arteries in symptomatic Caucasians and Chinese using vessel wall MRI.

Methods Subjects with recent anterior circulation ischaemic stroke were recruited and imaged at two sites in the USA and China using similar protocols. Both extracranial carotid and intracranial arteries were reviewed to determine plaque location and characteristics. Results The prevalence of extracranial carotid plaque in Caucasians and Chinese was $73.1 \%$ and $49.1 \%$, respectively $(p=0.055)$. Prevalence of intracranial plaque was $38.5 \%$ and $69.1 \%$ in Caucasians and Chinese, respectively ( $p=0.02)$. Furthermore, $42 \%$ of Caucasians and $16 \%$ of Chinese had high-risk plaque (HRP) features (intraplaque haemorrhage, luminal surface disruption) in the extracranial carotid artery $(p=0.03)$. The prevalence of HRP features in intracranial arteries was not significantly different between the two cohorts ( $4 \%$ vs $11 \% ; p=0.42$ ). Conclusions Differences in the location and characteristics of cerebrovascular atherosclerosis were identified by vessel wall MRI in US Caucasian and Chinese subjects with recent anterior circulation ischaemic stroke. Extracranial carotid plaques with HRP features were more common in Caucasians. Intracranial plaques were more common in Chinese subjects, but no significant difference between the two cohorts in intracranial HRP prevalence was found. Larger studies using vessel wall imaging to investigate racial differences in cerebrovascular disease may inform underlying mechanisms of HRP development and may ultimately help guide appropriate therapy.

\section{INTRODUCTION}

Stroke is the fifth and first leading cause of mortality in the USA and China, respectively. ${ }^{12}$ Atherosclerosis involving the extracranial carotid and intracranial arteries is an important contributor for ischaemic stroke. The location of cerebrovascular stenosis has been shown to differ between racial groups; in particular, extracranial carotid artery stenosis is more common among Caucasians while intracranial artery stenosis is more common among Asians. ${ }^{3-9}$ However, the differences in atherosclerotic plaque features besides luminal stenosis, and the variation between races in those characteristics based on plaque location have not yet been extensively examined. ${ }^{10}{ }^{11}$ Information on these differences may provide insight into variation in stroke risk in different racial groups.

Vessel wall MRI has proved capable of imaging the characteristics of extracranial carotid and intracranial atherosclerotic plaques in vivo. ${ }^{12-18}$ Multicontrast-weighted vessel wall MRI can reliably characterise plaque components such as intraplaque haemorrhage (IPH), lipid-rich necrotic core (LRNC), calcification and luminal surface condition. The presence of IPH and luminal surface disruption (eg, fibrous cap rupture or ulceration) on MRI is the hallmark of high-risk plaque (HRP), and has been shown in multiple prospective studies to be associated with a higher risk of future ischaemic stroke. ${ }^{19-22}$ Ideally, treatment of a patient with ischaemic stroke should be guided by knowledge of the location of the HRP within the extracranial carotid and intracranial arteries for effective future stroke prevention.

To our knowledge, simultaneous study of plaque components in both extracranial and intracranial cerebrovascular arteries has not been evaluated between Caucasians and Asians using vessel wall MRI. Therefore, our aim was to determine the distribution of atherosclerosis and investigate the presence of HRP features in both the extracranial carotid and intracranial arteries in Caucasians and Chinese with recent anterior circulation ischaemic stroke using vessel wall MRI, and assess plaque location and characteristic differences between the two racial groups. 


\section{METHODS}

\section{Study population}

The Culprit Plaque in Acute Cerebral Infarction Study is a cross-sectional, observational study jointly funded by the National Institutes of Health/NINDS (R01 NS083503), and the National Natural Science Foundation of China (83161120402). Serial patients 50-80 years old with anterior circulation (carotid territory) cerebral hemispheric ischaemic stroke within the prior 6 months were prospectively recruited from the University of Washington Medical Center and Affiliated Hospitals in the USA, and the Beijing Tiantan Hospital in China. Exclusion criteria were (1) inability to provide informed consent; (2) likely cardioembolic source for stroke (eg, atrial fibrillation); (3) contraindication to MRI; (4) estimated Glomerular Filtration Rate (eGFR) $<45 \mathrm{~mL} / \mathrm{min} / 1.73 \mathrm{~m}^{2} ;(5)$ history of allergy to gadolinium contrast; (6) prior carotid or intracranial arterial surgery or stenting; (7) history of radiation therapy to the head and neck; (8) pregnancy; (9) history of hepatorenal syndrome; and (10) history of liver transplantation. In this substudy, non-Caucasian subjects recruited from the US site were excluded from this analysis.

Demographic information and a detailed medical history were collected using questionnaires that were standardised across the two sites. Demographic characteristics including age, sex, height and weight were recorded. History of hypertension, hyperlipidaemia, diabetes mellitus, coronary heart disease and smoking (current or former) and medications were collected. The questionnaires and blood tests were obtained during their hospital visit. Institutional review board approvals were obtained for the entire study and from each participating institution, and all study participants provided written informed consent (IRB approval number: 46509-A in the USA and KY2014-004-04 in China).

\section{Magnetic resonance imaging}

Extracranial carotid and intracranial MRI protocols were implemented and standardised for plaque imaging across USA and Chinese sites. All MRIs were performed on 3.0T MR scanners, including Philips Achieva whole-body scanners (Philips Medical Systems, Best, Netherlands) at the University of Washington in the USA and Tsinghua University in China, and Siemens Magnetom Tim Trio (Siemens Medical Solution, Erlangen, Germany) at the Beijing Tiantan Hospital in China. Extracranial carotid and intracranial vessel wall MRI was performed using head coils for intracranial vessel wall MRI with either separate carotid coils for carotid vessel wall MRI or integrated with the head coil as a combined brain-carotid neurovascular array. ${ }^{23}$ Three different contrast-weighted images of the extracranial carotid and intracranial arteries were obtained: (1) T1W VISTA/MERGE ${ }^{12}$ or T1W SPACE, (2) simultaneous non-contrast angiography and IPH image ${ }^{13}$ or MPRAGE ${ }^{18}$ for IPH detection and (3) Time-of-Flight (TOF) MRA. The protocol was optimised to maintain similar scanner, coil and sequences at the three sites.

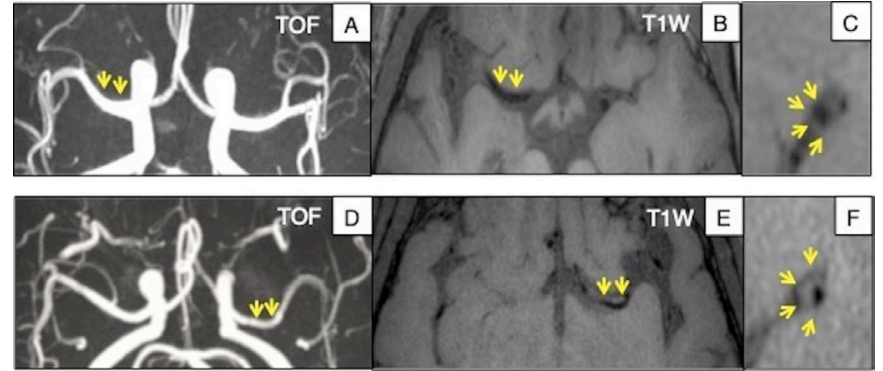

Figure 1 Example of intracranial MRI in USA and China. Upper row: Caucasian subject, (A) TOF MIP image shows no significant stenosis in the MCA. $(B)$ and $(C)$ Axial and sagittal views of 3D-T1 VISTA image shows no wall thickness. Bottom row: Chinese subject, (D) TOF MIP image shows no significant stenosis in the MCA. (E) and (F) Axial and sagittal views of 3D-T1 SPACE image reveals extraluminal hyperintensity of MCA segment, indicating plaque without significant stenosis. MCA, middle cerebral artery; MIP, maximum intensity projection.

The imaging protocol and parameters for this study are detailed in online supplementary table I.

\section{Image review}

Plaque detection and characterisation of extracranial carotid and intracranial artery disease on the symptomatic side was performed in a core lab (Vascular Imaging Lab, University of Washington, Seattle, USA) by an experienced reader with $>4$ years of experience interpreting carotid and intracranial vessel wall images using RadiAnt open-source DICOM viewer software (Medixant; Poznan, Portland). Each plaque component definition using a previously described multicontrast review approach ${ }^{14}$ is in online supplementary table II. In addition, 10 cases (five cases each from USA and China) from this study were randomly selected to be reviewed independently by a second reader who had $>10$ years of experience to assess inter-reader reproducibility. Readers were blinded to demographic, clinical and site (USA/China) information during image review. A plaque was identified as presence of focal wall thickening greater than or equal to 1.5 $\mathrm{mm}$ on an axial view in the extracranial carotid artery, ${ }^{24}$ and by apparent focal wall thickening in the intracranial artery (figures 1 and 2). ${ }^{17}$

For the extracranial carotid artery, the region $1 \mathrm{~cm}$ proximal and $1 \mathrm{~cm}$ to the common carotid bifurcation on the symptomatic side was reviewed. For the intracranial arteries, the following segments on the symptomatic side were reviewed: the cavernous and supraclinoid segments of the intracranial internal carotid artery, the A1 and A2 segments of the anterior cerebral artery and the M1 and M2 segments of the middle cerebral artery. Image quality was rated in each extracranial carotid and intracranial artery on a four-point scale: $1=$ arterial wall and vessel margins are unidentifiable, $2=$ arterial wall is visible, but the structure, lumen and outer boundaries are less distinct, $3=$ wall structures are identifiable, but lumen and outer boundaries are partially obscured, 4=vessel wall, 

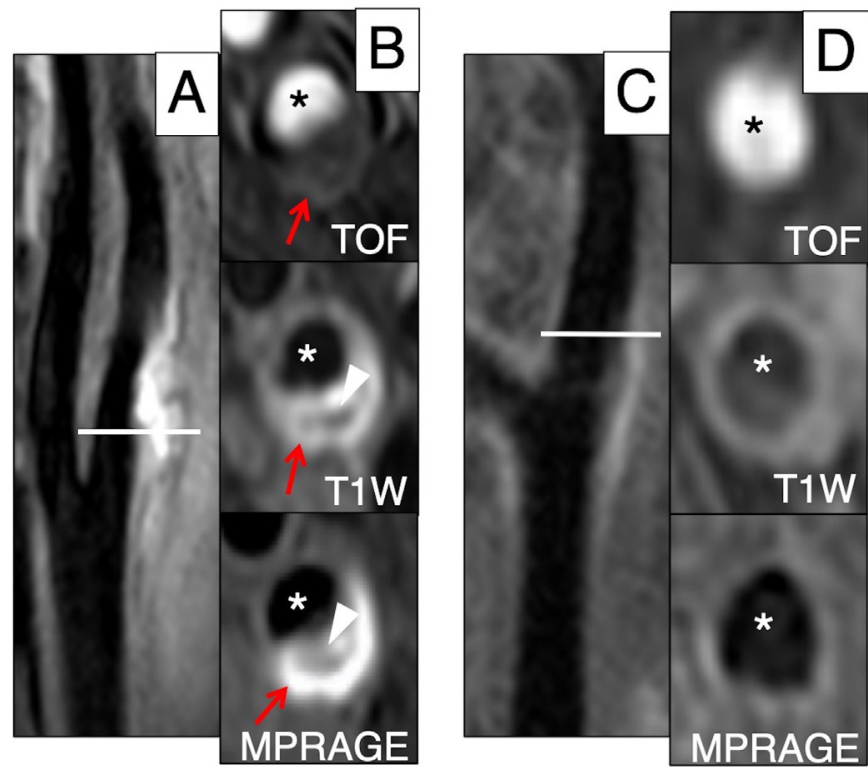

Figure 2 Example of extracranial carotid MRI in USA and China. Left: Caucasian subject, (A) Longitudinal view of extracranial carotid artery. The horizontal line indicates the level of the transverse carotid plaque images (shown in B). (B) The high-intensity area on TOF, T1W and MPRAGE indicates regions of IPH (red arrow). The low-intensity area on T1W and MPRAGE indicates a LRNC (arrowhead). Right: Chinese subject, $(C)$ longitudinal view of extracranial carotid artery. The horizontal line indicates the level of the transverse carotid plaque images (shown in D). (D) TOF shows no significant stenosis and T1W and MPRAGE show no plaque. Asterisk represents lumen. IPH, intraplaque haemorrhage; LRNC, lipid-rich necrotic core.

lumen and plaque components are well defined. Images with an image quality $<2$ were excluded from the study. The presence or absence of LRNC, IPH, calcification and luminal surface disruption including fibrous cap rupture and ulcer was recorded using previously published criteria (online supplementary table II). ${ }^{14-16}$ The same criteria were also adapted for intracranial artery plaque. HRP features were defined as presence of IPH or luminal surface disruption. Three-dimensional TOF MRA images were reconstructed by maximum intensity projection to identify presence of luminal stenosis of extracranial carotid and intracranial arteries.

\section{Statistical analysis}

The summary statistics of the data are presented as median with range for continuous variables and count with percentage for categorical variables. Clinical and plaque characteristics were compared between Caucasians and Chinese using the Mann-Whitney test for continuous variables or Fisher's exact test for categorical variables. Logistic regression was used to explore the impact of adjusting the association between race and HRP features for clinical risk factors. Due to the small sample size, clinical risk factors were adjusted for one-at-a-time in separate models. Reproducibility between two readers were assessed by calculating per

\begin{tabular}{|c|c|c|c|}
\hline & $\begin{array}{l}\text { Caucasians } \\
(n=26)\end{array}$ & $\begin{array}{l}\text { Chinese } \\
(n=55)\end{array}$ & $P$ value \\
\hline Age, years & $62(36-100)$ & 57 (39-79) & 0.09 \\
\hline Sex, male & $16(61.5)$ & $42(76.4)$ & 0.19 \\
\hline BMI, kg/m² & $27(17-40)$ & 24 (18-34) & 0.002 \\
\hline Current smoker & 5 (19.2) & $26(47.3)$ & 0.03 \\
\hline Hypertension & $18(69.2)$ & 37 (67.3) & $>0.99$ \\
\hline $\mathrm{sBP}, \mathrm{mm} \mathrm{Hg}$ & 133 (109-183) & $150(110-191)$ & 0.02 \\
\hline $\mathrm{dBP}, \mathrm{mm} \mathrm{Hg}$ & $84(65-107)$ & $90(60-124)$ & 0.01 \\
\hline Hyperlipidaemia & $20(80.0)$ & $21(38.2)$ & 0.001 \\
\hline $\mathrm{TC}, \mathrm{mg} / \mathrm{dL}$ & 168 (98-225) & $159(40-241)$ & 0.12 \\
\hline LDL, mg/dL & $90(47-162)$ & $98(34-172)$ & 0.96 \\
\hline HDL, mg/dL & $46(28-77)$ & $39(24-68)$ & 0.01 \\
\hline $\mathrm{TG}, \mathrm{mg} / \mathrm{dL}$ & $131(32-326)$ & $106(35-410)$ & 0.45 \\
\hline Diabetes & $4(15.4)$ & 18 (33.3) & 0.11 \\
\hline $\begin{array}{l}\text { Coronary heart } \\
\text { disease }\end{array}$ & $14(53.9)$ & $8(14.6)$ & $<0.001$ \\
\hline $\begin{array}{l}\text { On statins prior to } \\
\text { recent symptoms }\end{array}$ & $12(46.2)$ & $13(24.1)$ & 0.07 \\
\hline
\end{tabular}

Values are median (range) or number (percentage).

Bold indicates $\mathrm{P}$-values $<0.05$.

$\mathrm{BMI}$, body mass index; dBP, diastolic blood pressure;

HDL, high-density lipoprotein cholesterol; LDL, low-density lipoprotein cholesterol; sBP, systolic blood pressure; TC, total cholesterol; TG, triglyceride.

cent agreement and Cohen's kappa. A two-sided p value $<0.05$ was considered statistically significant, without adjustment for the number of tests. All analyses were performed using Stata/SE V.15.1 software (StataCorp; College Station, Texas, USA).

\section{RESULTS}

\section{Baseline characteristics in USA and China cohorts}

A total of 27 qualifying Caucasians in the USA and 155 qualifying Chinese in China were considered for this analysis. One case from the USA and 18 cases from China were excluded due to insufficient image quality. Of the remaining 137 Chinese, 55 subjects were selected following matching by sex and age $( \pm 5$ years $)$ to have a balanced comparison between Caucasians and Chinese. The characteristics of the subjects subgrouped by race are shown in table 1 . The Caucasian group had higher BMI, lower current smoking rate and lower systolic and diastolic blood pressure than the Chinese cohort. Also, Caucasians had a significantly higher prevalence of hyperlipidaemia $(80.0 \%$ vs $38.2 \%$, p value $=0.001)$ and coronary heart disease history $(53.9 \%$ vs $14.6 \%$, p value $<0.001)$, and higher levels of high-density lipoprotein cholesterol, compared with the Chinese. Otherwise, there were no significant differences in clinical characteristics between the two cohorts. 
Table 2 Comparison of plaque prevalence and features between Caucasians and Chinese

\begin{tabular}{|c|c|c|c|c|c|c|}
\hline & \multicolumn{3}{|c|}{ Extracranial carotid artery } & \multicolumn{3}{|c|}{ Intracranial artery } \\
\hline & $\begin{array}{l}\text { Caucasians } \\
(n=26)\end{array}$ & $\begin{array}{l}\text { Chinese } \\
(n=55)\end{array}$ & $P$ value & $\begin{array}{l}\text { Caucasians } \\
(n=26)\end{array}$ & Chinese $(n=55)$ & $P$ value \\
\hline Plaque & $19(73.1)$ & $27(49.1)$ & 0.055 & $10(38.5)$ & $38(69.1)$ & 0.02 \\
\hline Presence of luminal stenosis & $15(57.7)$ & $20(36.4)$ & 0.09 & $11(42.3)$ & $40(72.7)$ & 0.01 \\
\hline Number of plaques per subject ${ }^{\star}$ & - & - & - & $1.7 \pm 0.7$ & $1.7 \pm 0.9$ & 0.85 \\
\hline \multicolumn{7}{|l|}{ Plaque features } \\
\hline LRNC & $16(61.5)$ & $23(41.8)$ & 0.15 & $2(7.7)$ & $17(30.9)$ & 0.03 \\
\hline $\mathrm{IPH}$ & $6(23.1)$ & $10(18.2)$ & 0.77 & $1(3.9)$ & $2(3.6)$ & $>0.99$ \\
\hline Calcification & $7(26.9)$ & $6(10.9)$ & 0.10 & 0 & $8(14.6)$ & 0.050 \\
\hline Luminal surface disruption & $9(34.6)$ & $2(3.6)$ & $<0.001$ & 0 & $4(7.3)$ & 0.30 \\
\hline HRP & $11(42.3)$ & $9(16.4)$ & 0.03 & $1(3.9)$ & $6(10.9)$ & 0.42 \\
\hline
\end{tabular}

Values are number (percentage) or mean \pm SD.

Bold indicates $P$-values $<0.05$.

${ }^{\star}$ Number of plaques was only assessed in intracranial artery.

HRP, high-risk plaque; IPH, intra-plaque haemorrhage; LRNC, lipid-rich necrotic core.

\section{Plaque location}

Twenty-four of 26 Caucasian subjects had plaque in either the ipsilateral extracranial carotid or intracranial arteries compared with 45 of 55 Chinese subjects $(92.3 \%$ vs $81.8 \%, \mathrm{p}=0.32)$. Caucasians were somewhat more likely to have ipsilateral extracranial carotid plaque than Chinese $(73.1 \%$ vs $49.1 \%, \mathrm{p}=0.055)$ and significantly less likely to have ipsilateral intracranial plaque $(38.5 \%$ vs $69.1 \%, \mathrm{p}=0.02$, table 2 ). The number of segments with intracranial plaques per subject did not differ significantly between the two groups (mean: 1.7 vs $1.7, \mathrm{p}=0.85$ ), when subjects had at least one ipsilateral intracranial plaque. Figure 3 shows the distribution of subjects when grouped by the presence/absence of ipsilateral extracranial carotid and intracranial plaque. Caucasians were

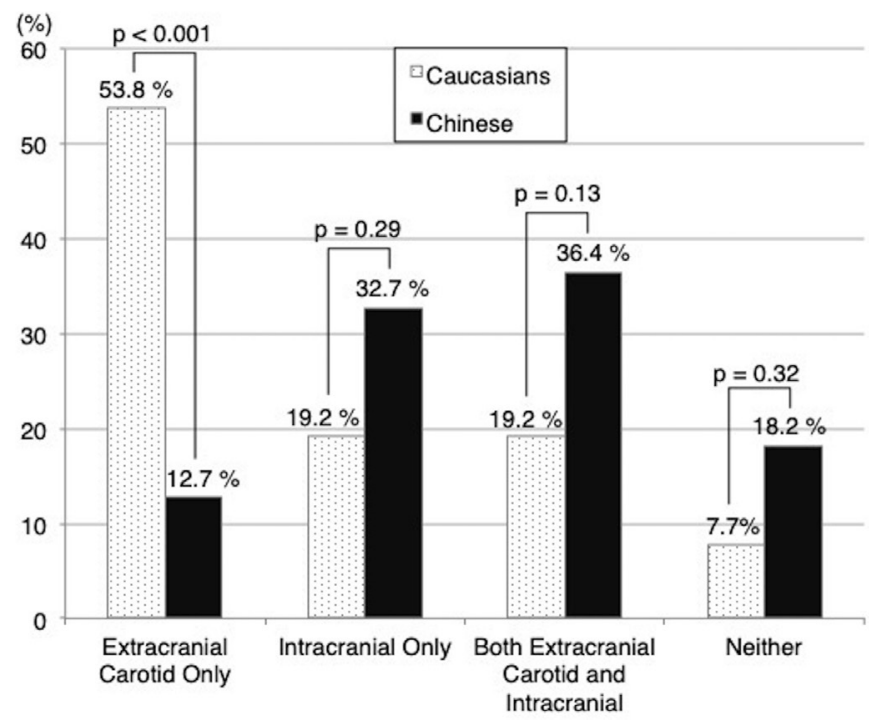

Figure 3 Comparison of plaque location between Caucasians and Chinese. $P$ value comparing Caucasian and Chinese using Fisher's exact test. significantly more likely than Chinese to have isolated ipsilateral extracranial carotid plaque without intracranial plaque $(53.8 \%(14 / 26)$ vs $12.7 \%(7 / 55), \mathrm{p}<0.001)$. In contrast, Caucasians less commonly had isolated intracranial plaque than Chinese, but this difference was not statistically significant $(19.2 \%(5 / 26)$ vs $32.7 \%$ $(18 / 55), \mathrm{p}=0.29$, figure 3$)$.

\section{Plaque features}

In the ipsilateral extracranial carotid artery, more Caucasian subjects had plaque with luminal surface disruption (34.6\% vs $3.6 \%, \mathrm{p}<0.001)$ and HRP $(42.3 \%$ vs $16.4 \%$, $\mathrm{p}=0.03$ ), compared with Chinese (table 2). However, the prevalence of LRNC $(61.5 \%$ vs $41.8 \%, \mathrm{p}=0.15)$, IPH (23.1\% vs $18.2 \%, \mathrm{p}=0.77)$ and calcification $(26.9 \%$ vs $10.9 \%, \mathrm{p}=0.10$ ) in the extracranial carotid arteries was not significantly different between Caucasians and Chinese. Fewer Caucasian than Chinese subjects had intracranial plaque with LRNC ( $7.7 \%$ vs $30.9 \%, \mathrm{p}=0.03)$. Other plaque features were found infrequently in the intracranial arteries of both races, including IPH $(3.9 \%$ vs $3.6 \%$, $\mathrm{p}>0.99$ ) and HRP (3.9\% vs $10.9 \%, \mathrm{p}=0.42$ ). None of the Caucasian subjects had calcification and luminal surface disruption in intracranial arteries, whereas $14.6 \%$ and $7.3 \%$ of the Chinese were found to have these features ( $\mathrm{p}=0.050$ and 0.30 , respectively).

When considering the presence of HRP in any artery, $42.3 \%(11 / 26)$ of the Caucasian and $27.3 \%(15 / 55)$ of the Chinese subjects had HRP in either extracranial carotid or intracranial arteries $(p=0.21)$. When further examined by location, HRP features were found only in the ipsilateral extracranial carotid artery in $10(38.5 \%)$ Caucasians and 9 (16.4\%) Chinese ( $\mathrm{p}=0.047)$, and only in the ipsilateral intracranial arteries in none of the Caucasians and in $6(10.9 \%)$ of the Chinese $(p=0.17$, figure 4). 


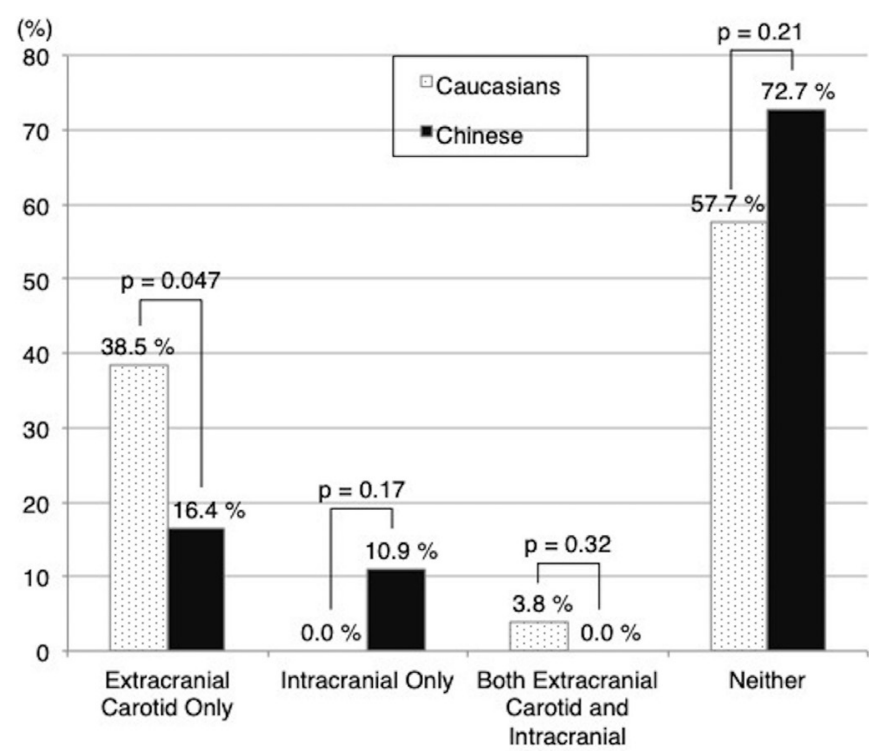

Figure 4 Comparison of location of HRP features between Caucasians and Chinese. $P$ value comparing Caucasian and Chinese using Fisher's exact test. HRP, high-risk plaque.

\section{Clinical risk factors for presence of HRP}

To determine whether clinical risk factors influence the presence of HRP, each clinical risk factor was compared between subjects with and without HRP features. Subjects with HRP features in the ipsilateral extracranial carotid artery tended to be older compared with those without HRP (62 years old (range: 50-83) and 58 years old (range: 36-100), respectively; $\mathrm{p}=0.051$, online supplementary table III). Before adjustment for clinical risk factors, race (Caucasian vs Chinese) was associated with an OR for extracranial carotid HRP of 3.75 (95\% CI: 1.30 to $10.78, \mathrm{p}=0.01$ ). Race remained statistically significant after adjusting each clinical risk factor individually, with resulting adjusted ORs ranging from 3.05 to 4.62 and corresponding $\mathrm{p}$ values $<0.055$ in all cases. HRP in intracranial arteries was not associated with race before and after adjustment for clinical risk factors (table 3 ).

Reproducibility of MRI to identify plaque location and components

Of the 10 cases selected for inter-reader reproducibility analysis, the two trained readers agreed on the presence or absence of plaque in the extracranial carotid arteries $80 \%$ of the time $(8 / 10)$, with a corresponding kappa of 0.55 (95\% CI: 0.04 to 1.00). Similarly, of the 60 intracranial segments reviewed (10 cases: 6 segments per case), the two readers agreed on the presence or absence of plaque in the intracranial arteries $85 \%$ of the time $(51 / 60)$, with a corresponding kappa of 0.65 (95\% CI: 0.43 to 0.87 ). With regard to plaque component detection including LRNC, IPH, calcification and luminal surface disruption, agreement between the two trained readers ranged from $60 \%$ to $100 \%$ of the 10 extracranial carotid arteries and $95 \%$ to $98 \%$ of the 60 intracranial segments.

\section{DISCUSSION}

To our knowledge, this is the first study to investigate not only plaque location, but also plaque features of both extracranial carotid and intracranial arteries using vessel wall MRI in Caucasians and Chinese with recent anterior circulation ischaemic stroke. Subjects in the USA and China were recruited using the same inclusion and exclusion criteria and imaged using the same or similar MRI protocol. Plaques in the ipsilateral extracranial carotid artery tended to be found more commonly in Caucasians than in the Chinese subjects. On the other hand, the prevalence of ipsilateral intracranial plaques in Caucasians was less than that in Chinese. A key finding of this study is that HRP features (IPH and luminal surface disruption) were markedly more prevalent in the extracranial carotid arteries of Caucasians compared with Chinese, and this difference did not appear to be explained by differences

Table 3 Presence of HRP for race and clinical factors

\begin{tabular}{|c|c|c|c|c|c|c|}
\hline & \multicolumn{3}{|c|}{ Extracranial carotid artery } & \multicolumn{3}{|c|}{ Intracranial artery } \\
\hline & OR & $95 \% \mathrm{Cl}$ & $P$ value & OR & $95 \% \mathrm{Cl}$ & $P$ value \\
\hline Caucasian vs Chinese & 3.75 & 1.30 to 10.78 & 0.01 & 0.33 & 0.04 to 2.86 & 0.31 \\
\hline \multicolumn{7}{|l|}{ Adjustment factor } \\
\hline Age, years & 3.05 & 1.01 to 9.21 & 0.047 & 0.31 & 0.03 to 2.89 & 0.30 \\
\hline Sex, male & 4.08 & 1.38 to 12.07 & 0.01 & 0.36 & 0.04 to 3.20 & 0.36 \\
\hline $\mathrm{BMI}, \mathrm{kg} / \mathrm{m}^{2}$ & 3.40 & 1.03 to 11.27 & 0.045 & 0.53 & 0.05 to 5.11 & 0.58 \\
\hline Current smoker & 4.00 & 1.31 to 12.23 & 0.02 & 0.23 & 0.03 to 2.06 & 0.19 \\
\hline Hypertension & 3.86 & 1.33 to 11.22 & 0.01 & 0.30 & 0.03 to 2.74 & 0.29 \\
\hline Hyperlipidaemia & 3.14 & 0.97 to 10.10 & 0.055 & 0.26 & 0.03 to 2.54 & 0.25 \\
\hline Diabetes & 3.31 & 1.13 to 9.69 & 0.03 & 0.36 & 0.04 to 3.24 & 0.36 \\
\hline Coronary heart disease & 4.62 & 1.40 to 15.19 & 0.01 & 0.17 & 0.02 to 1.79 & 0.14 \\
\hline On statins prior to recent symptoms & 3.48 & 1.18 to 10.26 & 0.02 & 0.21 & 0.02 to 2.02 & 0.18 \\
\hline
\end{tabular}

Bold indicates $\mathrm{P}$-values $<0.05$.

$\mathrm{BMI}$, body mass index; HRP, high-risk plaque. 
in any particular clinical risk factor. However, the prevalence of HRP features in the intracranial arteries was not significantly different between Caucasians and Chinese.

\section{Location of extracranial carotid and intracranial plaques}

Many studies have reported racial differences for extracranial carotid and intracranial artery atherosclerotic stenosis. Caucasians have more extracranial carotid artery stenosis, whereas Asians, African-Americans and Hispanics have been reported to have more intracranial artery stenosis using angiography or ultrasound. $.^{4-8} 25-27$ These are similar with our results, where $57.5 \%$ of Caucasians and $36.4 \%$ of Chinese had luminal stenosis on 3D TOF MRA in the extracranial carotid arteries, and $42.3 \%$ and $72.7 \%$ in the intracranial arteries. In addition, our study found that extracranial carotid plaque on vessel wall MRI tended to be more prevalent in Caucasians than in Chinese, and intracranial plaque was significantly less prevalent in Caucasians than in Chinese (table 2).

Although we found that the number of plaques in intracranial arteries per subject was not significantly different between Caucasians and Chinese, Hao et al found that non-Caucasians, including Asians, AfricanAmericans and Hispanics had a higher number of intracranial atherosclerotic plaques, compared with Caucasians using vessel wall MRI. ${ }^{11}$ A possible reason why our findings are inconsistent with Hao $e t$ al is that the latter reviewed all intracranial arteries of subjects with stroke for the presence of stenosis, whereas we reviewed only the symptomatic side of the anterior intracranial arteries in subjects with anterior circulation stroke. Further, the presence of carotid or intracranial stenosis was not one of the inclusion criteria for our study. The extracranial carotid artery is morphologically and embryologically distinct from the intracranial arteries $^{2628} 29$ and the haemodynamic flow conditions differ between the two vascular beds. ${ }^{30} 31$ Thus, the atherosclerotic process may differ between extracranial carotid and intracranial arteries. Differences in the prevalence of certain risk factors may contribute to the different location of cerebrovascular atherosclerosis. ${ }^{32}$ As the history of hyperlipidaemia was more prevalent in Caucasians compared with Chinese, hyperlipidaemia may be a factor favouring extracranial carotid atherosclerosis. ${ }^{33}$ In addition, there may be differences in genetic susceptibility, but it has been difficult to dissociate these effects. The definitive reasons why plaque location varies between races remain uncertain.

\section{Plaque features of extracranial carotid and intracranial arteries}

We previously found that Caucasians had a smaller volume of LRNC and more calcified extracranial carotid plaque compared with Chinese on vessel wall MRI among subjects with carotid-related neurological symptoms and $>50 \%$ stenosis. ${ }^{10}$ In contrast, we did not find a significant difference in the prevalence of LRNC and calcified plaque between Caucasians and Chinese in the current study (table 2). This disparity is likely due to different inclusion criteria between the two studies; the presence of carotid or intracranial stenosis was not required for entry in this study. The Chinese cohort in our study tended to have less severe or no plaque in the extracranial carotid artery, whereas all Chinese subjects in the prior study had $>50 \%$ carotid stenosis.

Previous autopsy studies have shown that $5.8 \%$ of Caucasian and $30.3 \%$ of Chinese subjects with stroke with $>30 \%$ intracranial artery stenosis had HRP features in intracranial arteries. ${ }^{34}{ }^{35}$ However, we did not find significant differences in HRP features of intracranial arteries between the two groups, though such features were found in $15 \%$ of subjects. As noted above, presence of carotid or intracranial stenosis was not an entry requirement for this study. An additional possible explanation is that some of the smaller, more distal lesions identified in postmortem histological studies are below the detection threshold of the intracranial MRI protocol utilised in our study.

\section{Clinical implications}

More precise identification of the aetiology of ischaemic stroke is critical for effective current stroke management and future stroke prevention, as the treatment options for culprit carotid and intracranial lesions differ. Further, a more comprehensive evaluation of both the extracranial- and intracranial cerebrovasculature may identify stroke aetiology in the approximately $30 \%$ of patients with cryptogenic stroke. ${ }^{36} 37$ Finally, a better understanding of racial differences of not only plaque distribution, but also plaque characteristics in patients with acute stroke may provide insights into regional environmental and genetic risk factors for the development of high-risk atheroma.

\section{LIMITATIONS}

The number of subjects in this study is relatively small. As such, these findings should be considered hypothesis generating and require confirmation in future larger studies. Second, both the USA and China recruitment sites are specialised stroke centres. As such, the findings from this study may not be representative of the generalised ischaemic stroke population. Third, intracranial MR vessel wall imaging is an emerging field with newer protocols under development that have higher spatial resolution and better image quality. ${ }^{38}$ Improvements afforded by contemporary state-of-the-art techniques may permit identification of intracranial HRP features that may be under the threshold for detection in the current study. Lastly, this study is cross-sectional in design. Prospective longitudinal studies are needed to further establish the clinical utility of cerebrovascular vessel wall imaging with MRI. 


\section{CONCLUSION}

Extracranial carotid plaques with MRI-detected HRP features are more common in US Caucasians than in Chinese subjects with recent anterior circulation ischaemic stroke. Intracranial plaques with a LRNC were more common in the Chinese cohort; however, no significant difference in the prevalence of intracranial HRP was found. These findings demonstrate the potential utility of vessel wall MRI to identify the location of culprit plaques responsible for ischaemic stroke, which will help guide appropriate therapy for secondary stroke prevention. Larger studies using vessel wall MRI to investigate these racial differences may also lead a better understanding of environmental and genetic factors leading to the development of HRP.

\section{Author affiliations}

${ }^{1}$ Department of Surgery, University of Washington, Seattle, Washington, USA ${ }^{2}$ Department of Radiology, Beijing Tiantan Hospital, Capital Medical University, Beijing, China

${ }^{3}$ Tiantan Neuroimaging Center for Excellence, Beijing Tiantan Hospital, Capital Medical University, Beijing, China

${ }^{4}$ Beijing Neurosurgical Institute, Beijing, China

${ }^{5}$ Department of Neurosurgery, Beijing Tiantan Hospital, Capital Medical University, Beijing, China

${ }^{6}$ Department of Radiology, University of Washington, Seattle, Washington, USA ${ }^{7}$ Department Medicine (Medical Genetics) and Genome Sciences, University of Washington, Seattle, Washington, USA

${ }^{8}$ Center for Biomedical Imaging Research, Department of Biomedical Engineering, Tsinghua University, Beijing, China

Acknowledgements We wish to thank the research participants who volunteered for this study, and the Bio-Molecular Imaging Center at the University of Washington for MR scanning services.

Contributors HW, BS, PG, ZD, GPJ, CY and TSH contributed to the study design. HW, MS, BS, JS, NB, XZ, RL and SC implemented the study. DSH supervised the statistical analyses. TSH and CY supervised the findings of this work. HW wrote the manuscript with support from TSH. All authors contributed to the final manuscript.

Funding This research received the National Institutes of Health (R01 NS083503) and the National Natural Science Foundation of China (83161120402). JS received support from the American Heart Association (17MCPRP33671077).

Competing interests None declared.

Patient consent for publication Not required.

Provenance and peer review Not commissioned; externally peer reviewed. Data availability statement Data are available upon reasonable request.

Open access This is an open access article distributed in accordance with the Creative Commons Attribution Non Commercial (CC BY-NC 4.0) license, which permits others to distribute, remix, adapt, build upon this work non-commercially, and license their derivative works on different terms, provided the original work is properly cited, appropriate credit is given, any changes made indicated, and the use is non-commercial. See: http://creativecommons.org/licenses/by-nc/4.0/.

ORCID iD

Hiroko Watase http://orcid.org/0000-0002-8951-7692

\section{REFERENCES}

1 Virani SS, Alonso A, Benjamin EJ, et al. Heart disease and stroke Statistics-2020 update: a report from the American heart association. Circulation 2020;141:CIR0000000000000757.

2 Wang $\mathrm{W}$, Jiang $\mathrm{B}$, Sun $\mathrm{H}$, et al. Prevalence, Incidence, and Mortality of Stroke in China: Results from a Nationwide Population-Based Survey of 480687 Adults. Circulation 2017;135:759-71.

3 Caplan LR, Gorelick PB, Hier DB. Race, sex and occlusive cerebrovascular disease: a review. Stroke 1986;17:648-55.
4 Wityk RJ, Lehman D, Klag M, et al. Race and sex differences in the distribution of cerebral atherosclerosis. Stroke 1996;27:1974-80.

5 Sacco RL, Roberts JK, Boden-Albala B, et al. Race-ethnicity and determinants of carotid atherosclerosis in a multiethnic population. the Northern Manhattan stroke study. Stroke 1997;28:929-35.

6 Kieffer SA, Takeya Y, Resch JA, et al. Racial differences in cerebrovascular disease. Angiographic evaluation of Japanese and American populations. Am J Roentgenol Radium Ther Nucl Med 1967;101:94-9.

7 Brust RW. Patterns of cerebrovascular disease in Japanese and other population groups in Hawaii: an angiographical study. Stroke 1975;6:539-42.

8 Nishimaru K, McHenry LC, Toole JF. Cerebral angiographic and clinical differences in carotid system transient ischemic attacks between American Caucasian and Japanese patients. Stroke 1984;15:56-9.

9 Sacco RL, Kargman DE, Gu Q, et al. Race-ethnicity and determinants of intracranial atherosclerotic cerebral infarction. The Northern Manhattan stroke study. Stroke 1995;26:14-20.

10 Saam T, Cai JM, Cai YQ, et al. Carotid plaque composition differs between ethno-racial groups: an MRI pilot study comparing mainland Chinese and American Caucasian patients. Arterioscler Thromb Vasc Biol 2005;25:611-6.

11 Hao Q, Qiao Y, Gottesman R, et al. Racial difference in intracranial atherosclerotic plaque features by high-resolution vessel wall MRI. 49. Los Angeles: International Stroke Conference, Stroke, 2018.

12 Balu N, Yarnykh VL, Chu B, et al. Carotid plaque assessment using fast 3D isotropic resolution black-blood MRI. Magn Reson Med 2011;65:627-37

13 Wang J, Börnert P, Zhao H, et al. Simultaneous noncontrast angiography and intraplaque hemorrhage (SNAP) imaging for carotid atherosclerotic disease evaluation. Magn Reson Med 2013;69:337-45.

14 Cai J-M, Hatsukami TS, Ferguson MS, et al. Classification of human carotid atherosclerotic lesions with in vivo multicontrast magnetic resonance imaging. Circulation 2002;106:1368-73.

15 Chu B, Kampschulte A, Ferguson MS, et al. Hemorrhage in the atherosclerotic carotid plaque: a high-resolution MRI study. Stroke 2004;35:1079-84.

16 Hatsukami TS, Ross R, Polissar NL, et al. Visualization of fibrous cap thickness and rupture in human atherosclerotic carotid plaque in vivo with high-resolution magnetic resonance imaging. Circulation 2000;102:959-64.

17 Mossa-Basha M, Hwang WD, De Havenon A, et al. Multicontrast high-resolution vessel wall magnetic resonance imaging and its value in differentiating intracranial vasculopathic processes. Stroke 2015;46:1567-73.

18 Hishikawa T, lihara K, Yamada N, et al. Assessment of necrotic core with intraplaque hemorrhage in atherosclerotic carotid artery plaque by MR imaging with 3D gradient-echo sequence in patients with high-grade stenosis. Clinical article. J Neurosurg 2010;113:890-6.

19 Takaya N, Yuan C, Chu B, et al. Association between carotid plaque characteristics and subsequent ischemic cerebrovascular events: a prospective assessment with MRI--initial results. Stroke 2006;37:818-23.

20 Saam T, Hetterich H, Hoffmann V, et al. Meta-analysis and systematic review of the predictive value of carotid plaque hemorrhage on cerebrovascular events by magnetic resonance imaging. J Am Coll Cardiol 2013:62:1081-91.

21 Hosseini AA, Kandiyil N, Macsweeney STS, et al. Carotid plaque hemorrhage on magnetic resonance imaging strongly predicts recurrent ischemia and stroke. Ann Neurol 2013;73:774-84.

22 Gupta A, Baradaran H, Schweitzer AD, et al. Carotid plaque MRI and stroke risk: a systematic review and meta-analysis. Stroke 2013;44:3071-7.

23 Wang X, Li R, Hayes C, et al. Yuan C. a new designed 36-Channel neurovascular coil at 3T. Proceedings of the 20th Scientific Meeting and Exhibition, International Society of Magnetic Resonance in Medicine, 2012.

24 Polak JF, Pencina MJ, Pencina KM, et al. Carotid-wall intimamedia thickness and cardiovascular events. $N$ Engl J Med 2011;365:213-21.

25 Feldmann E, Daneault N, Kwan E, et al. Chinese-white differences in the distribution of occlusive cerebrovascular disease. Neurology 1990;40:1540-5.

26 Inzitari D, Hachinski VC, Taylor DW, et al. Racial differences in the anterior circulation in cerebrovascular disease. How much can be explained by risk factors? Arch Neurol 1990;47:1080-4.

27 Wang MY, Mimran R, Mohit A, et al. Carotid stenosis in a multiethnic population. J Stroke Cerebrovasc Dis 2000;9:64-9. 
28 Nakamura M, Ishihara Y, Sata T, et al. Acid mucopolysaccharides and lipids of Japanese arteries, with special reference to the absence of hyaluronic acid in Japanese cerebral artery. $J$ Atheroscler Res 1966;6:132-50.

29 Menshawi K, Mohr JP, Gutierrez J. A functional perspective on the embryology and anatomy of the cerebral blood supply. J Stroke 2015;17:144-58.

30 Lindegaard KF, Bakke SJ, Grolimund P, et al. Assessment of intracranial hemodynamics in carotid artery disease by transcranial Doppler ultrasound. J Neurosurg 1985;63:890-8.

31 Nicholls SC, Phillips DJ, Primozich JF, et al. Flow separation in the carotid bulb: prognostic significance. Neurol Res 1992;14:85-8.

$32 \mathrm{Kim}$ JS, Nah H-W, Park SM, et al. Risk factors and stroke mechanisms in atherosclerotic stroke: intracranial compared with extracranial and anterior compared with posterior circulation disease. Stroke 2012;43:3313-8

$33 \mathrm{Kim}$ BJ, Kim JS. Ischemic stroke subtype classification: an Asian viewpoint. J Stroke 2014;16:8-17.
34 Mazighi M, Labreuche J, Gongora-Rivera F, et al. Autopsy prevalence of intracranial atherosclerosis in patients with fatal stroke. Stroke 2008;39:1142-7.

35 Chen XY, Wong KS, Lam WWM, et al. Middle cerebral artery atherosclerosis: histological comparison between plaques associated with and not associated with infarct in a postmortem study. Cerebrovasc Dis 2008;25:74-80.

36 Yaghi S, Bernstein RA, Passman R, et al. Cryptogenic stroke: research and practice. Circ Res 2017;120:527-40.

37 Li L, Yiin GS, Geraghty OC, et al. Incidence, outcome, risk factors, and long-term prognosis of cryptogenic transient ischaemic attack and ischaemic stroke: a population-based study. Lancet Neurol 2015;14:903-13.

38 Mandell DM, Mossa-Basha M, Qiao Y, et al. Intracranial vessel wall MRI: principles and expert consensus recommendations of the American Society of Neuroradiology. AJNR Am J Neuroradiol 2017;38:218-29. 